\title{
ALOCAÇÃO DOS CUSTOS DE MELHORIAS EM BACIAS HIDROGRÁFICAS ENTRE OS USUÁRIOS DE ÁGUA: UMA APLICAÇÃO DO VALOR DE SHAPLEY ${ }^{1}$
}

\author{
Gil Bracarense Leite ${ }^{2}$ \\ Wilson da Cruz Vieira ${ }^{3}$
}

Resumo: Este trabalho objetivou analisar a alocação dos custos de melhorias em bacias hidrográficas entre os diferentes demandantes de água. Tomou-se como exemplo a bacia do rio Paraíba do Sul, localizada nos estados de Minas Gerais, São Paulo e Rio de Janeiro, e utilizaram-se, como critérios de alocação de custos, os volumes de água captada e consumida, além da quantidade lançada de efluentes. A metodologia utilizada consistiu da regra de alocação conhecida como valor de Shapley, pertencente ao ramo cooperativo da teoria dos jogos. No caso da bacia do rio Paraíba do Sul, os valores encontrados indicaram que os usuários urbanos deveriam arcar com a maior parte dos custos, já que este é o setor que mais lança efluentes, sendo a deterioração da qualidade da água um problema importante nesta bacia.

Palavras-chave: recursos hídricos, alocação de custos, valor de Shapley, bacia do rio Paraíba do Sul.

\section{Introdução}

A água é um dos recursos naturais mais importantes do Planeta. Porém, a diversificação e a expansão da atividade econômica, ocorridas nas últimas décadas, fizeram com que muitos países identificassem problemas relativos à qualidade de suas águas, o que tem gerado redução na sua

\footnotetext{
Recebido em 20/02/2009; Aceito em 30/04/2009. Parte da dissertação de mestrado do primeiro autor, que foi selecionada para receber o Prêmio Edward Schuh (Melhor Dissertação de Mestrado em Economia), no $47^{\circ}$. Congresso da SOBER, a ser realizado em julho de 2009, em Porto Alegre - RS. Este artigo foi também submetido e aprovado para apresentação no referido Congresso.

2 Economista, Mestre em Economia Aplicada pela Universidade Federal de Viçosa. Viçosa - MG. E-mail: gilbracarense@yahoo.com.br.

3 Professor Associado do Departamento de Economia Rural da Universidade Federal de Viçosa. CEP 36570000 Viçosa - MG. E-mail: wvieira@ufv.br.
} 
disponibilidade (CAMPOS, 2001). Além disso, a distribuição geográfica dos recursos hídricos é bastante desigual, o que, conforme Tundisi (2003), combinado com a precária administração ambiental e com o desperdício no seu uso, tem feito com que esse bem se torne cada vez mais escasso até em áreas bem providas de água doce.

Nesse contexto, é cada vez mais iminente o surgimento de conflitos entre usuários, uma vez que a utilização de água tem objetivos diversos e, muitas vezes, excludentes, entre os quais se destacam a agricultura, pesca, indústria, saneamento urbano, turismo, proteção ambiental, navegação, etc. Dessa forma, segundo Carrera-Fernandez e Ferreira (2002), se medidas urgentes não forem adotadas, a escassez dos recursos hídricos poderá fazer com que esses conflitos pelo uso se tornem irremediáveis e comprometam o desenvolvimento de certas regiões. Torna-se necessário, portanto, adotar instrumentos eficientes de gestão que busquem uma alocação mais adequada e justa da água, entre seus múltiplos usos.

No Brasil, conforme preceitos estabelecidos pela legislação (BRASIL, 1997), a gestão das águas deve ocorrer no âmbito das bacias hidrográficas, sendo essas, portanto, as unidades administrativas dos recursos hídricos ${ }^{4}$. A lei designou, ainda, a criação de comitês, que são os órgãos responsáveis pela gestão dentro de cada bacia.

Os comitês de bacia hidrográfica são formados por representantes do poder público; por usuários das águas na sua área de atuação; e por entidades civis de recursos hídricos com atuação comprovada na bacia. O número de representantes de cada setor, bem como os critérios para sua indicação, deve ser estabelecido nos regimentos internos de cada comitê. Entre as diversas competências que lhes são atribuídas para tratar de questões relevantes em sua área de atuação, destacam-se (BRASIL, 1997):

4 A implantação desse novo sistema de gestão dos recursos hídricos foi estabelecida na Constituição brasileira de 1988, e a lei federal sobre o tema - "Lei das Águas" (nº 9433), foi sancionada em 1997. 
i. promover o debate das questões relacionadas com recursos hídricos;

ii. arbitrar, em primeira instância administrativa, os conflitos relacionados com os recursos hídricos;

iii. aprovar o Plano de Recursos Hídricos da bacia;

iv. estabelecer mecanismos de cobrança pelo uso de recursos hídricos e sugerir os valores a serem cobrados; e

v. estabelecer critérios e promover o rateio de custo das obras de uso múltiplo, de interesse comum ou coletivo.

As melhorias (estudos, projetos, etc.), incluídas no Plano de Recursos Hídricos para uma bacia hidrográfica, conforme atribuições acima, devem ser financiadas por recursos arrecadados dos usuários de água. Desse modo, uma questão iminente e possível fonte de conflitos referentes à gestão de recursos hídricos é o fato de como os custos de melhorias de interesse coletivo, que ocorrerão no interior de uma bacia, serão alocados entre os principais usuários.

Nesse sentido, este trabalho objetivou analisar a alocação de custos (entre os demandantes de água) dos múltiplos projetos de melhoria que podem ser implantados em uma bacia. Para isso, propôs-se a metodologia do valor de Shapley que é uma regra de alocação da teoria dos jogos que se adapta aos critérios estabelecidos pela legislação brasileira e é, em sua essência, uma metodologia justa, em que o sentido de justiça relacionase ao fato de que os demandantes de água são cobrados de acordo com o grau em que utilizam os recursos hídricos.

A bacia do rio Paraíba do Sul - uma das mais importantes do território nacional - serviu de fonte de dados para a consecução deste trabalho. Uma vez que o surgimento dos comitês e o estabelecimento da cobrança pelo uso da água são relativamente recentes no Brasil, espera-se contribuir para as discussões sobre este tema, tendo em vista o gerenciamento eficiente dos recursos hídricos. 


\section{Caracterização da Bacia do Rio Paraíba do Sul ${ }^{5}$}

A bacia do rio Paraíba do Sul (BRPS) está localizada na região Sudeste do Brasil, em três dos principais estados do país: São Paulo, Minas Gerais e Rio de Janeiro, com área de, aproximadamente, $55.500 \mathrm{~km}^{2}$, conforme mostra a Figura 1. Em sua extensão, encontram-se, atualmente, 180 municípios, e a população total, estimada para o ano de 2005 , foi de cerca de 5,35 milhões de habitantes.

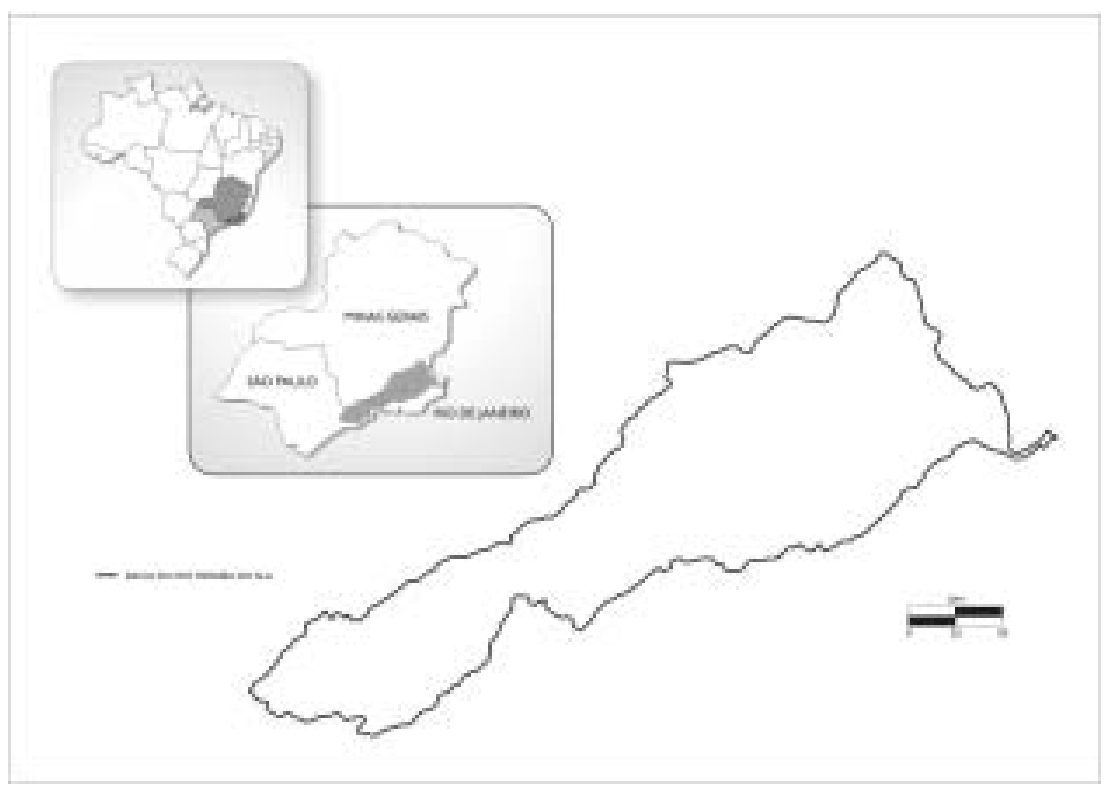

Figura 1. Localização geográfica da bacia do rio Paraíba do Sul.

A região onde se localiza a BRPS é uma das mais importantes no cenário econômico brasileiro, visto que abriga importantes indústrias e centros

\footnotetext{
${ }^{5}$ Esta seção está baseada em COPPETEC (2007) e Formiga-Johnsson et al. (2007).
} 
urbanos que respondem por quase $10 \%$ da riqueza gerada no país. A maior parte das atividades econômicas desenvolvidas na bacia está relacionada às formas de uso urbano e industrial, fazendo com que mais de $90 \%$ da população se concentre em áreas urbanas.

A principal fonte de preocupação na bacia é o declínio na qualidade da água devido à poluição industrial e, principalmente, aos esgotos municipais que não são tratados ${ }^{6}$. Ao longo do rio Paraíba do Sul e de seus afluentes, indústrias se instalaram e cidades cresceram, lançando efluentes nos rios, na maioria das vezes sem qualquer tratamento, degradando a qualidade de suas águas e reduzindo sua disponibilidade.

O órgão gestor dos recursos hídricos na BRPS é o CEIVAP (Comitê para Integração da Bacia Hidrográfica do Rio Paraíba do Sul), instituído em 1996. O CEIVAP foi um dos pioneiros na implantação no Brasil da cobrança pelo uso da água em 2003. A fórmula da cobrança, adotada inicialmente pelo CEIVAP, tem a seguinte interpretação: o comitê estabeleceu um preço unitário $\left(\mathrm{R} \$ / \mathrm{m}^{3}\right)$ para captação de água feita por cada um dos principais usuários (urbanos, industriais, agricultores, mineração, entre outros). Esse preço serviu de base para o cálculo da cobrança, sendo modificado de acordo com coeficientes específicos relacionados com uso de água de cada um dos setores demandantes ${ }^{7}$.

Essa fórmula vigorou na bacia no período de 2003 a 2006, sendo útil na comparação com a fórmula proposta neste trabalho, já que ambas têm o propósito de criar uma taxa para os usuários. A mais recente metodologia de cobrança pelo uso da água na bacia baseia-se em preços estabelecidos para o tipo de uso (captação, consumo e diluição de efluentes) e não para os usuários.

Concentrando-se nos usuários mais relevantes (agricultura, indústria e abastecimento urbano), tem-se que as quantidades captada e consumida

\footnotetext{
Apenas 17,6\% das regiões urbanas da BRPS tratam seus efluentes domésticos.

7 Para uma descrição detalhada da fórmula de cobrança pelo uso da água na bacia do rio Paraíba do Sul recomenda-se consultar o site http://www.ceivap.org.br.
} 
de água, além do lançamento de efluentes na BRPS, seguem o padrão apresentado na Tabela 1, em estimativas realizadas para o ano de 2005.

Tabela 1 - Captação de água, consumo de água e lançamento de efluentes por usuário na bacia do rio Paraíba do Sul, ano de 2005

\begin{tabular}{lcccccc}
\hline \multirow{2}{*}{ Usuário } & \multicolumn{2}{c}{ Captação } & \multicolumn{2}{c}{ Consumo } & \multicolumn{2}{c}{ Lançamento } \\
\cline { 2 - 7 } & $\left(\mathrm{m}^{3} / \mathrm{s}\right)$ & $(\%)$ & $\left(\mathrm{m}^{3} / \mathrm{s}\right)$ & $(\%)$ & $(\mathrm{kg} / \mathrm{s})$ & $(\%)$ \\
\hline 1. Indústria & 13,66 & 16,11 & 6,21 & 14,86 & 0,46 & 13,92 \\
2. Urbano & 17,99 & 21,21 & 3,60 & 8,61 & 2,85 & 86,08 \\
3. Agricultura & 53,16 & 62,68 & 31,99 & 76,53 & 0 & 0 \\
\hline Total & 84,81 & 100,00 & 41,80 & 100,00 & 3,31 & 100,00 \\
\hline
\end{tabular}

Fonte: COPPETEC (2007a).

Em relação ao lançamento de efluentes, o único parâmetro poluente considerado é a demanda bioquímica de oxigênio (DBO) já que, segundo Campos (2001), as informações disponíveis não possibilitam a estimativa de outros poluentes.

\section{Referencial Teórico}

Os agentes econômicos têm a possibilidade de interagirem, estrategicamente, em ampla variedade de formas, e grande parte delas vem sendo estudada por meio do ferramental da teoria dos jogos (VARIAN, 1992). Desde seu desenvolvimento formal, a partir da segunda metade do século passado, a teoria dos jogos tem ganhado cada vez mais importância e novas abordagens têm possibilitado sua aplicação em áreas diversas, não se restringindo apenas à economia. A definição formal de um jogo pode ser encontrada em Myerson (1991), que o trata como uma representação de uma situação em que os agentes (jogadores), ao agirem de maneira racional, interagem dentro de um cenário de interdependência estratégica, de modo que, para tomar decisão, cada agente leva em conta também as ações dos outros jogadores. 
Os jogos estratégicos, que envolvem dois ou mais jogadores, são divididos em não-cooperativos e cooperativos, sendo este último a teoria na qual se baseia o presente trabalho. $\mathrm{O}$ aspecto central que caracteriza os jogos cooperativos é a formação de coalizões por grupos de jogadores. $\mathrm{Na}$ maioria das vezes, as coalizões factíveis e o conjunto viável de pagamentos (payoffs) disponível para seus membros são dados, de modo que a questão a ser tratada é a identificação dos pagamentos finais recebidos por cada jogador, ou seja, dada uma coleção de conjuntos viáveis de payoffs, um para cada coalizão, tenta-se prever ou recomendar o valor ou conjunto de valores destinados a cada jogador (SERRANO, 2007).

Podem-se utilizar diferentes formas para representar um jogo. Em um jogo cooperativo, todavia, a representação mais comumente usada é na forma de função característica, apresentada, a seguir, junto a outras definições que caracterizam um jogo nesse formato. Seja $N=\{1, \ldots, n\}$ um conjunto finito de jogadores e uma coalizão, cada subconjunto nãovazio de $N$. Refere-se ao conjunto $N$, composto por todos os jogadores, como a grande coalizão. Pode-se especificar, para cada coalizão $C$, um conjunto $v(C) \subseteq \mathfrak{R}^{|C|}$ que contém vetores de pagamentos de $|C|$ dimensões, que são viáveis para a coalizão $C$. O conjunto $v(C)$ é a função característica, e o par $(N, v)$ representa um jogo cooperativo. Essa formulação considera que as ações tomadas pela coalizão complementar (os jogadores em $N \backslash C$ ) não tenham poder para interferir nas escolhas de $C$ (ALIPRANTIS; CHAKRABARTI, 2000).

Considerando a função característica $v(C)$, seus vetores podem ser apresentados com base em diversos conceitos de solução, porém, de acordo com Myerson (1991), na maioria dos casos, a solução sustentase na forma da grande coalizão, em que a cooperação total é alcançada. Uma solução deve designar um conjunto de vetores em $v(N)$, para cada jogo cooperativo $(N, v)$. Quando o objetivo é encontrar uma solução 
cooperativa na forma de conjunto de valores, o Core é o principal conceito, enquanto o valor de Shapley é o conceito mais usado quando a solução for um valor único, sendo este último o utilizado neste trabalho.

Nas questões abordadas na teoria dos jogos cooperativos, o Core é tratado como uma solução positiva, visto que, se um payoff não pertencer ao Core, ele não será um resultado viável. Já o valor de Shapley é um conceito de solução normativa, o que indica que a quantia que um jogador recebe (ou paga) é determinada por sua contribuição aos grupos aos quais ele pertence (SERRANO, 2007).

O interesse no qual se ateve Shapley (1953) é a resolução de um modo justo e com valor único de um problema de alocação (de custos, de benefícios, etc.) entre os agentes que fazem parte de um jogo, quando se leva em consideração o valor de cada coalizão $C$. O valor de $C$, representado por $v(C)$, expressa a posição inicial da coalizão - o quanto ela tem à sua disposição. Shapley propôs, então, um método de solução que passou a se chamar valor de Shapley. Conforme Aliprantis e Chakrabarti (1999), este tem sido usado como regra de alocação em ampla variedade de contextos, servindo para analisar contendas em áreas tão díspares quanto gestão de recursos hídricos, alocação de impostos e taxas de aterrissagem em aeroportos.

O valor de Shapley é uma regra $\Phi$, que associa a cada jogo de $n$ pessoas, $\quad N=\{1,2, \ldots, n\}$, um vetor de $n$ dimensões $\Phi(v)=\left(\Phi_{1}(v), \Phi_{2}(v), \ldots, \Phi_{n}(v)\right)$, que deve satisfazer às seguintes propriedades (ALIPRANTIS; CHAKRABARTI, 2000): 
i. $\quad$ Eficiência: $\sum_{i=1}^{n} \Phi_{i}(v)=v(N)$;

ii. Simetria: Para qualquer permutação ${ }^{8} \pi$ de $v$ e cada jogador $i$ tem-se $\Phi_{\pi(i)}(\pi v)=\Phi_{i}(v)$. Tal relação significa que o valor $\Phi_{i}(v)$ não depende do rótulo do jogador, mas da sua posição (ordenamento) no jogo em relação à função característica $v$;

iii. Linearidade: Se $u$ e $v$ são dois jogos quaisquer de $n$ pessoas e $\alpha$ e $\beta$ são escalares, então $\Phi(\alpha u+\beta v)=\alpha \Phi(u)+\beta \Phi(v)$, em que $\alpha u+\beta v$ denota um jogo de $n$ pessoas para $(\alpha u+\beta v)(C)=\alpha u(C)+\beta v(C) ; \mathrm{e}$

iv. Irrelevância de jogadores $d u m m y^{9}$ : Se $i$ for um jogador dummy, então $\Phi_{i}(v)=0$.

Estabelecidas essas propriedades, Shapley argumentou que a classe de todos os jogos nesse formato tem um único valor, o valor de Shapley, que designa pagamentos dados pelos componentes $\Phi(v)=\left(\Phi_{1}(v), \Phi_{2}(v), \ldots, \Phi_{n}(v)\right)$ para cada jogador $i$, apresentado a seguir:

$$
\begin{aligned}
& \Phi_{i}(v)=\sum_{C \subseteq N \mid\{i\}} \frac{|C| !(|N|-|C|-1) !}{|N| !}[v(C \cup\{i\})-v(C)] \text {, para cada } \\
& i=1, \ldots, n
\end{aligned}
$$

\footnotetext{
${ }^{8}$ Uma permutação $\pi$ de jogadores é simplesmente uma função um a um $\pi: N \rightarrow N$, que representa, tão-somente, um rearranjo de jogadores.

9 Um jogador $i$ é dito irrelevante (jogador dummy) $v(C \cup\{i\})=v(C)$ se se sustentar em toda coalizão, ou seja, um jogador dummy não acrescenta nada ao associar-se a qualquer coalizão.
} 
em que $|N|$ é o total de jogadores; $|C|$ designa o número de jogadores na coalizão $C$; e a expressão $v(C \cup\{i\})-v(C)$ representa o valor marginal do jogador $i$, quando ele se associa à coalizão $C$.

Por considerar todas as associações possíveis, o valor de Shapley de um jogo normalmente é visto como uma regra de alocação que dá a cada jogador a média do valor marginal que ele adiciona, unindo-se a uma coalizão. Além disso, o valor de Shapley é considerado uma regra de alocação justa, onde o conceito de justiça não é o de equidade e, sim, o conceito de que a quantia que os participantes de um jogo pagam (ou recebem) é determinada por sua contribuição. Se for esperado que um indivíduo acrescente pouco (muito) a uma coalizão, então a quantia alocada a ele pelo valor de Shapley tende a ser pequena (grande) (SERRANO, 2007).

\section{Modelo Analítico}

\subsection{Valor de Shapley aplicado ao uso de recursos hídricos}

O valor de Shapley pode ser aplicado ao uso dos recursos hídricos por meio de uma adaptação de exemplo presente em Aliprantis e Chakrabarti (2000, pp. 228$)^{10}$. Para a aplicação proposta, utilizaram-se como critérios de alocação de custos entre os usuários de água as quantidades captada e consumida de água e o lançamento de efluentes por cada um dos usuários. Ao considerar esses três critérios, objetivou-se dar importância tanto ao aspecto quantitativo quanto qualitativo no uso dos recursos hídricos. O desenvolvimento da aplicação foi feito inicialmente para a captação de água, porém, construção análoga será adotada adiante para os outros critérios.

\footnotetext{
${ }^{10}$ Exemplos de aplicação do valor de Shapley para alocação de custos entre os usuários de recursos hídricos podem ser vistos em Loehman et al. (1979) e Young et al. (1982).
} 
As pressuposições da aplicação proposta foram as seguintes. Considerou-se que cada usuário de água pode arcar, individualmente, com o custo das melhorias que lhe atenderá ou financiá-las conjuntamente com outros usuários. Ao financiar, em separado, esse custo, o usuário pagará um montante que é proporcional à quantidade que ele capta de água ${ }^{11}$. Admitiu-se que o custo total das melhorias é equivalente ao custo incorrido pelo usuário que capta a maior quantidade de água se este arcasse sozinho com as melhorias relacionadas à sua atividade de demandante. O custo total é a quantia a ser dividida, caso todos os usuários agissem em conjunto para disponibilizar as melhorias.

Considerou-se $K_{t}$ o custo incorrido para atender, individualmente, ao usuário do tipo $t$, em que $t=1, \ldots, T$, ou seja, $K_{t}$ é o custo que o usuário tem para financiar as melhorias que lhe atenderá, sem se unir aos outros usuários. Admitiu-se que $0<K_{1}<K_{2}<\ldots<K_{T}$, isto é, quanto maior a captação de água, maior será o custo das melhorias para aquele usuário de água.

A suposição de que o custo total das melhorias, $K_{T}$, é equivalente ao custo do usuário que apresenta os maiores índices para o critério em consideração, caso ele financie sozinho as melhorias, indica a presença de economias de escala na formação de coalizões para o financiamento conjunto dos custos. As economias de escala são necessárias para a cooperação ocorrer, do contrário, esta não seria vantajosa para os jogadores.

O número de jogadores pode ser analisado pelo volume de água estimado para ser captado por cada usuário, em certo período de tempo. Sob essa ótica, os jogadores são identificados pela quantidade de água captada

\footnotetext{
${ }_{11}$ Reitera-se que a aplicação desenvolvida a seguir foi feita considerando somente o critério da quantidade captada de água. Por isso, nessa suposição, o custo é proporcional à captação de água. Quando forem levados em consideração os três critérios - captação, consumo e lançamento de efluentes - o custo será proporcional aos três.
} 
por cada um ${ }^{12}$. Uma coalizão $C$, nesse jogo, é um subconjunto de $N=\{1,2, \ldots, n\}$. Considerando-se que $N_{t}$ denota o conjunto do volume de água captado por um usuário do tipo $t$, fica claro que $N=U_{t=1}^{T} N_{t}$ e $N_{t} \cap N_{s}=\varnothing$ para $t \neq s$. Além disso, tem-se que, para cada coalizão $C, t(C)=\max \left\{t \in\{1,2, \ldots, T\}: C \cap N_{t} \neq \varnothing\right\}$, ou seja, $t(C)$ é o usuário que capta a maior quantidade de água e, portanto, é o que incorre em maiores custos dentro da coalizão $C$. Com isso, pode-se definir a função característica $v$ do jogo por $v(C)=-K_{t(C)}$, isto é, o valor da coalizão é equivalente ao maior custo entre seus membros. Pode-se perceber que $v(N)=-K_{T}$, o que significa que o custo total é coberto pelas quantias cobradas de cada usuário.

Para um jogo com essas características, segundo Aliprantis e Chakrabarti (2000), a fórmula usual do valor de Shapley, dada pela equação (1), é pouco prática. Adotou-se uma abordagem apresentada por esses autores e que é descrita a seguir. Inicialmente, define-se o conjunto:

$$
A_{\ell}=\bigcup_{t=\ell}^{T} N_{t}
$$

Definem-se, também, $T$ jogos de $n$ jogadores com funções características $v_{1}, \ldots, v_{T}$, dadas por

$$
v_{\ell}(C)=\left\{\begin{array}{l}
0, \text { se } C \cap A_{\ell}=\varnothing \\
K_{\ell-1}-K_{\ell}, \text { se } C \cap A_{\ell} \neq \varnothing .
\end{array}\right.
$$

\footnotetext{
12 Um exemplo numérico pode facilitar a compreensão: considere que o volume total de água captado em uma bacia seja de $80 \mathrm{~m}^{3} / \mathrm{s}$. Supõe-se que existam três usuários de água que captam essa quantidade total, da seguinte forma: $15 \mathrm{~m}^{3} / \mathrm{s}$ para o usuário $1 ; 25 \mathrm{~m}^{3} / \mathrm{s}$ para o usuário 2 ; e $40 \mathrm{~m}^{3} / \mathrm{s}$ para o usuário 3 . Assim, pela observação do volume captado de água, pode-se identificar esses três "tipos" de jogadores. Se um jogador capta $15 \mathrm{~m}^{3} /$ $\mathrm{s}$ de água, então ele é tratado como um jogador do tipo 1 ; se capta $25 \mathrm{~m}^{3} / \mathrm{s}$ é do tipo 2 ; e se ele capta $40 \mathrm{~m}^{3} / \mathrm{s}$ é um jogador do tipo 3 .
} 
O valor da cada coalizão $C$ é $v(C)=\sum_{\ell=1}^{T} v_{\ell}(C)$. Para perceber isso, nota-se que, se $\ell \leq t(C)$, então $C \cap A_{\ell} \neq \varnothing$, enquanto se $\ell>t(C)$, então $C \cap A_{\ell}=\varnothing$. Assim,

$\sum_{\ell=1}^{T} v_{\ell}(C)=\sum_{\ell=1}^{t(C)}\left(K_{\ell-1}-K_{\ell}\right)=K_{0}-K_{t(C)}=v(C)$,

em que $K_{0}=0$, ou seja, se não há jogadores, não há custos.

Sabe-se que, pela propriedade aditiva ${ }^{13}$ do valor de Shapley,

$$
\varphi(v)=\sum_{\ell=1}^{T} \varphi\left(v_{\ell}\right)
$$

Deve-se, então, computar $\varphi_{i}\left(v_{\ell}\right)$, o valor de Shapley, para cada jogador $i$. Primeiramente, percebe-se que, pela definição de $v_{\ell}$, segue-se que:

$$
v_{\ell}(C \cup\{i\})-v_{\ell}(C)=\left\{\begin{array}{l}
K_{\ell-1}-K_{\ell}, \quad \text { se } C \cap A_{\ell}=\varnothing \text { e } i \in A_{\ell} \\
0, \text { caso contrário }
\end{array}\right.
$$

Desse modo, para cada $i \in A_{\ell}$, o valor de Shapley é dado por

$$
\varphi_{i}\left(v_{\ell}\right)=\sum_{C \subseteq N \mid A_{\ell}} \frac{|C| !(|N|-|C|-1) !}{|N| !}\left(K_{\ell-1}-K_{\ell}\right) \text {. }
$$

$\overline{{ }^{13} \forall S, T \subset N, S \cap T} T=\varnothing$ então $v(S \cup T) \geq v(S)+v(T)$. 
Em particular, tem-se que $\varphi_{i}\left(v_{\ell}\right)=\varphi_{j}\left(v_{\ell}\right)$, para todo $i, j \in A_{\ell}$. Para todo $i \notin A_{\ell}$, tem-se $\varphi_{i}\left(v_{\ell}\right)=0$. Então,

$\left(\sum_{t=\ell}^{T}\left|N_{t}\right|\right) \varphi_{i}\left(v_{\ell}\right)=\sum_{i \in A_{\ell}} \varphi_{i}\left(v_{\ell}\right)=v_{\ell}(N)=K_{\ell-1}-K_{\ell}$.

Conseqüentemente,

$\varphi_{i}\left(v_{\ell}\right)=\frac{K_{\ell-1}-K_{\ell}}{\sum_{t=\ell}^{T}\left|N_{t}\right|}$.

Segue-se que, para todo $i$ e $\ell$, o valor de Shapley para o jogo $v_{\ell}$ satisfaz a seguinte relação:

$\varphi_{i}\left(v_{\ell}\right)=\left\{\begin{array}{l}0, \text { se } i \notin A_{\ell} \\ \frac{K_{\ell-1}-K_{\ell}}{\sum_{t=\ell}^{T}\left|N_{t}\right|}, \quad \text { se } \quad i \in A_{\ell}\end{array}\right.$

Recordando que $\varphi(v)=\sum_{\ell=1}^{T} \varphi_{i}\left(v_{\ell}\right)$ e que $i \in N_{k}$ implica $i \in A_{\ell}$ para $\ell \leq k$, obtém-se a expressão que é o valor de Shapley do jogo para cada participante da coalizão, considerando apenas o critério da quantidade captada de água, ou seja,

$\varphi_{i}(v)=\sum_{\ell=1}^{k} \frac{K_{\ell-1}-K_{\ell}}{\sum_{t=\ell}^{T}\left|N_{t}\right|}, \quad i \in N_{k}, \quad k=1,2, \ldots, T$. 
Pode-se encontrar expressão equivalente à equação (2) para os critérios do consumo de água e da quantidade lançada de efluentes por meio de desenvolvimento idêntico ao que resultou na equação citada e seguindo exatamente as mesmas pressuposições. Obtém-se, assim, o valor de Shapley, $\varphi_{i}^{C}(v)$, para o critério do consumo de água:

$$
\varphi_{i}^{C}(v)=\sum_{\ell=1}^{k^{C}} \frac{K_{\ell-1}^{C}-K_{\ell}^{C}}{\sum_{t=\ell}^{T}\left|N_{t}^{C}\right|}, \quad i \in N_{K^{C}}^{C}, \quad k^{C}=1,2, \ldots, T .
$$

Por sua vez, o valor de Shapley, $\varphi_{i}^{L}(v)$, quando a alocação de custos tem como base o critério da quantidade lançada de efluentes, é dado por:

$$
\varphi_{i}^{L}(v)=\sum_{\ell=1}^{k^{L}} \frac{K_{\ell-1}^{L}-K_{\ell}^{L}}{\sum_{t=\ell}^{T}\left|N_{t}^{L}\right|}, \quad i \in N_{K^{L}}^{L}, \quad k^{L}=1,2, \ldots, T .
$$

Porém, alocar os custos considerando cada critério separadamente não seria interessante já que os três são importantes atributos de uso da água. Portanto, propôs-se a junção das equações (2), (3) e (4), obtendose o valor de Shapley do jogo, $\Phi_{i}(v)$, como uma combinação convexa dos três critérios apresentados e que foi aplicado na alocação dos custos da implantação de melhorias na bacia do rio Paraíba do Sul, ou seja,

$$
\Phi_{i}(v)=\alpha \times \varphi_{i}(v)+\beta \times \varphi_{i}^{C}(v)+(1-\alpha-\beta) \times \varphi_{i}^{L}(v),
$$

em que $\alpha, \beta$ e $(1-\alpha-\beta)$ são ponderações, com $\alpha$ e pertencentes ao intervalo $[0,1]$, respeitando a restrição $\alpha+\beta \leq 1$. Esses parâmetros indicam o peso relativo de cada critério em determinada bacia 
A representação de cada critério junto a uma ponderação traz a possibilidade de que se construam diferentes cenários, alterando estes pesos de acordo com características específicas da bacia hidrográfica que estiver sendo estudada. Desse modo, adotando-se $\alpha+\beta=1$, considera-se que a bacia esteja limpa e, no cálculo do valor de Shapley, a importância total será destinada aos critérios captação e consumo de água, ou seja, projetos que assegurem volumes de água para atender essas demandas específicas na bacia. No entanto, ao se adotar $\alpha=\beta=0$, ter-se-á o caso de uma bacia poluída, e o critério lançamento de efluentes será o único considerado no cálculo do valor de Shapley, ou seja, projetos para despoluir a bacia seriam prioritários.

Na identificação dos jogadores, a aplicação restringiu-se aos setores industrial, urbano e agrícola que são os mais relevantes, visto que respondem por quase a totalidade da quantidade demandada de água na BRPS. Os outros usuários têm impacto limitado no uso da água e, por isso, foram tratados como jogadores irrelevantes.

\section{Resultados e Discussão}

Nesta seção utilizaram-se dados referentes aos usuários de água da BRPS para a aplicação do valor de Shapley ponderado, considerando três diferentes cenários: uma bacia limpa, uma bacia poluída (cenários extremos) e um cenário intermediário que está próximo à realidade da bacia. Como tal aplicação gera resultados em que a cobrança é estabelecida para cada usuário de água, pode-se compará-los com os preços que eram a base da metodologia de cobrança que vigorou na bacia no período 2003-2006 e que tinha o mesmo aspecto, qual seja, o estabelecimento de um preço para cada usuário, sendo que este preço servia de base para a cobrança ${ }^{14}$.

${ }_{14}$ Por razões de praticidade, utilizou-se a metodologia de cobrança antiga já que esta pode ser comparada diretamente com o resultado do valor de Shapley. Porém, a metodologia em vigência atualmente (desde 2007) também pode ser comparada com o valor de Shapley, necessitando, todavia, que se realize antes da comparação uma adaptação em sua fórmula. 
Em relação ao valor a ser alocado entre os usuários - que representa os custos das supostas melhorias - utilizou-se a arrecadação potencial ao longo de um ano, que equivale à $\mathrm{R} \$ 23.087 .612,82$. Este valor foi encontrado aplicando-se a própria fórmula de cobrança que vigorava na BRPS, mas, considerando que todos os usuários de água na bacia pagariam para usá-la. Sua vantagem é que, ao utilizá-la na aplicação do valor de Shapley, os resultados deste tornam-se diretamente comparáveis com os valores cobrados na bacia. Utilizando, portanto, a arrecadação potencial na bacia ao longo de um ano (em $\mathrm{R} \$$ ) e o volume estimado de água captado por cada setor em um ano $\left(\mathrm{em} \mathrm{m}^{3}\right)$, o valor de Shapley encontrará uma taxa em $\mathrm{R} \$ / \mathrm{m}^{3}$ para a cobrança pelo uso da água em determinado ano. Com essas considerações iniciais, pode-se iniciar os cálculos necessários para a obtenção dos resultados.

O custo total anual das melhorias representado pelo potencial arrecadável com a cobrança $(\mathrm{R} \$ 23.087 .612,82)$ pode ter sua estrutura de alocação, sob cada um dos três critérios considerados (captação, consumo e lançamento), representada por meio de índices que variam de 0 a 100. Esses índices possibilitam a generalização da aplicação, pois o resultado final mostra-se na forma de porcentagem do custo destinado a cada usuário, de modo que qualquer valor de custo que for proposto pode ser inserido na fórmula, para que sua alocação seja encontrada.

Respeitando as relações de proporção existentes, os índices que representam a alocação de custo das melhorias para cada usuário, considerando que a alocação será feita separadamente, de acordo com os critérios de captação, consumo e lançamento de efluentes, são apresentados na Tabela 2. Os índices - conforme as suposições do desenvolvimento realizado na seção 4.1 - indicam a parte percentual do custo total incorrida por cada demandante, se este arcasse individualmente com o ônus das melhorias que lhe atenderiam, em vez de realizá-las junto aos outros usuários. 
Tabela 2 - Índice de custo das melhorias para cada usuário, pelos critérios de captação de água, consumo de água e lançamento de efluentes

\begin{tabular}{lccc}
\hline Usuário & $\begin{array}{c}\text { Índice de Custo: } \\
\text { Captação }\end{array}$ & $\begin{array}{c}\text { Índice de Custo: } \\
\text { Consumo }\end{array}$ & $\begin{array}{c}\text { Índice de Custo: } \\
\text { Lançamento de efluentes }\end{array}$ \\
\hline 1. Indústria & 25,70 & 19,41 & 16,17 \\
2. Urbano & 33,84 & 11,25 & 100,00 \\
3. Agrícola & 100,00 & 100,00 & - \\
\hline
\end{tabular}

Fonte: Resultados da pesquisa.

Para o critério da captação de água, por exemplo, se suportasse sozinho o custo das melhorias, o usuário industrial teria um ônus proporcional à própria quantidade captada de água, o que equivale a $25,70 \%$ do custo a ser alocado. Outra suposição é de que o custo total das melhorias seria equivalente ao custo incorrido pelo usuário que capta a maior quantidade de água, caso este financiasse as melhorias sem se unir aos outros usuários. Desse modo, ainda para o critério da captação, se o usuário agrícola não se associasse a outros usuários, ele arcaria com um valor igual a 100\% do custo total a ser alocado - ou seja, o exato valor da arrecadação potencial de $\mathrm{R} \$ 23.087 .612,82$ - para financiar as melhorias ${ }^{15}$. Como delineado ao desenvolver a aplicação, esta suposição é uma forma de justificar a existência de economias de escala na formação de coalizões.

Portanto, o setor agrícola, por ser aquele que capta a maior quantidade de água, teria de arcar com um valor equivalente ao total do custo, caso pagasse, em separado, pelas melhorias que lhe trariam benefícios. Os setores urbano e industrial, que captam quantidades menores de água, pagariam uma parte menor do custo total, se cada um arcasse individualmente com os custos dos projetos relacionados ao uso da água para captação. Raciocínio semelhante pode ser feito para justificar os índices de custo pelos critérios do consumo de água e lançamento de efluentes.

\footnotetext{
$\overline{15}$ Não se deve confundir, neste ponto, a ordem dos acontecimentos. A suposição apresentada não significa que o usuário que não se associar será responsável pelo custo total das intervenções na bacia. A interpretação correta é que se os jogadores não se associarem cada um terá um custo individual. Porém, se todos se associarem para dividir os custos de forma conjunta, eles terão - devido às economias de escala - um custo total que é igual ao custo que o maior usuário teria, caso agisse individualmente.
} 
No período de um ano, para o qual se deseja alocar o custo das melhorias, o volume esperado de água demandada para captação e consumo, bem como a quantidade esperada para o lançamento de efluentes, estão na Tabela 3. Contudo, como os três critérios são representados por unidades de medidas diferentes, foram criados novamente índices, representando os valores percentuais, que visam tornar possível o cálculo do valor de Shapley ponderado, em que é necessário somar os três critérios.

Tabela 3 - Estimativas anuais para os volumes captado e consumido de água e para a quantidade lançada de efluentes por cada usuário na bacia do rio Paraíba do Sul

\begin{tabular}{lcccccc}
\hline Usuário & $\begin{array}{c}\text { Volume captado } \\
\left(\mathrm{m}^{3} / \mathrm{ano}\right)\end{array}$ & $(\%)$ capt. & $\begin{array}{c}\text { Volume } \\
\text { consumido } \\
\left(\mathrm{m}^{3} / \mathrm{ano}\right)\end{array}$ & $\begin{array}{c}(\%) \text { cons. } \\
\text { Quant. lançada } \\
(\mathrm{kg} / \mathrm{ano})\end{array}$ & $\begin{array}{c}(\%) \\
\text { lanç. }\end{array}$ \\
\hline 1. Ind. & 430.781 .760 & 16,11 & 195.838 .560 & 14,86 & 14.537 .950 & 13,92 \\
2. Urb. & 567.332 .640 & 21,21 & 113.529 .600 & 8,61 & 89.917 .750 & 86,08 \\
3. Agr. & 1.676 .453 .760 & 62,68 & 1.008 .836 .640 & 76,53 & - & - \\
\hline Total & 2.674 .568 .160 & 100 & 1.318 .204 .800 & 100 & 104.455 .700 & 100 \\
\hline
\end{tabular}

Fonte: Resultados da pesquisa.

Com os dados apresentados nas Tabelas 2 e 3, pode-se computar o valor de Shapley para os usuários, considerando cada um dos critérios isoladamente, usando as equações (2), (3) e (4), que possibilitam que se proceda, em seguida, ao cálculo ponderado. A Tabela 4 exemplifica os valores das cobranças realizadas para cada usuário considerando, separadamente, cada um dos critérios. Como o valor de Shapley foi construído com base nos índices que retiraram a unidade de medida dos três critérios e do custo, os valores ainda não têm uma interpretação direta, já que não estão representados como unidades monetárias. 
Tabela 4 - Valor de Shapley calculado para os critérios de captação e consumo de água e lançamento de efluentes para a bacia do rio Paraíba do Sul

\begin{tabular}{lccc}
\hline Usuário & $\begin{array}{c}\text { Valor de Shapley: } \\
\text { Captação }\end{array}$ & $\begin{array}{c}\text { Valor de Shapley: } \\
\text { Consumo }\end{array}$ & $\begin{array}{c}\text { Valor de Shapley: } \\
\text { Efluentes }\end{array}$ \\
\hline 1. Indústria & 0,2570 & 0,2018 & 0,1617 \\
2. Urbano & 0,3540 & 0,1125 & 1,1355 \\
3. Agricultura & 1,4095 & 1,2548 & - \\
\hline
\end{tabular}

Fonte: Resultados da pesquisa.

Os valores presentes na Tabela 4 foram utilizados nas seções que se seguem para encontrar a taxa cobrada de cada usuário, em que o cálculo do valor de Shapley foi ponderado pelos três critérios, utilizando a equação (5) e levando em conta a existência dos três diferentes cenários sugeridos para a BRPS: uma bacia limpa, uma bacia poluída - que são os cenários extremos - e um cenário intermediário.

Para cada usuário, multiplicando seus respectivos valores de Shapley (Tabela 4) pelas porcentagens que representam a captação, o consumo e o lançamento de efluentes (Tabela 3 ) e pelos valores das ponderações $\alpha$ e $\beta$ escolhidos em cada cenário, encontra-se a porcentagem do custo total que lhe deve ser atribuída. Com isso, tem-se, para cada cenário, a parte de cada usuário referente ao custo dos múltiplos projetos que podem ser desenvolvidos em uma bacia. Finalmente, com este último valor e com a quantidade captada de água por cada usuário ao longo de um ano, pode-se encontrar uma taxa medida em $\mathrm{R} \$ / \mathrm{m}^{3}$, tornando possível a comparação com o preço-base que vigorou na BRPS até 2006.

\subsection{Cenário da bacia limpa $(\alpha+\beta=1)$}

Em uma situação hipotética para a BRPS, em que a poluição das águas fosse desprezível, a única fonte de preocupação seria o caráter quantitativo da água. Como conseqüência, a melhoria a ser financiada pelos usuários estaria ligada, por exemplo, a projetos que proporcionassem o aumento da oferta de água ou o seu melhor aproveitamento. 
Uma vez que a preocupação nesse cenário é somente com a água em seu aspecto quantitativo, importância total no cálculo da taxa cobrada dos usuários deveria ser reservada aos critérios das quantidades captada e consumida de água. Atribui-se peso maior ao consumo $(\beta=0,6)$ devido ao maior impacto que este causa nos corpos d'água em comparação à captação $(\alpha=0,4)$. Percebe-se, pela observação da Tabela 5, que o usuário que é o principal demandante de água (setor agrícola), teria de arcar com uma parcela maior dos custos das melhorias e pagar a taxa mais elevada.

Tabela 5 - Alocação dos custos e taxas cobradas dos usuários, num cenário em que a bacia do rio Paraíba do Sul estivesse limpa

\begin{tabular}{lccc}
\hline Usuário & $\%$ do Custo & $(\%) \times 23.087 .612,82(\mathrm{R} \$)$ & Taxa $\left(\mathrm{R} \$ / \mathrm{m}^{3}\right)$ \\
\hline 1. Indústria & 3,45 & $823.877,38$ & 0,0018 \\
2. Urbano & 3,59 & $978.840,81$ & 0,0015 \\
3. Agrícola & 92,96 & $21.284 .894,63$ & 0,0128 \\
\hline Total & 100,00 & $23.087 .612,82$ & - \\
\hline
\end{tabular}

Fonte: Resultados da pesquisa.

Se o lançamento de efluentes em suas águas não fosse um problema, os setores potencialmente poluidores - industrial e urbano - seriam beneficiados e pagariam taxas de $0,0018 \mathrm{R} \$ / \mathrm{m}^{3}$ e $0,0015 \mathrm{R} \$ / \mathrm{m}^{3}$, que representam, respectivamente, $14,46 \%$ e $11,40 \%$ do valor cobrado dos usuários agrícolas para prover as melhorias, com custo anual de R $\$$ 23.087.612,82. Do valor total, o setor industrial pagaria 3,45\% do custo dos projetos desenvolvidos, enquanto os usuários urbanos cobririam 3,59\%, o que indica que, se a BRPS tivesse essas características, haveria alocação de custo favorável aos dois setores citados. O setor agrícola, pelo contrário, ao pagar uma taxa de $0,0128 \mathrm{R} \$ / \mathrm{m}^{3}$, ficaria responsável por parcela significativa dos custos, cobrindo um montante que equivaleria a 92,96\% do custo total dos projetos relacionados ao consumo e à captação de água. 


\subsection{Cenário da bacia poluída ( $\boldsymbol{\alpha}=\boldsymbol{\beta}=\mathbf{0})$}

Numa situação para a BRPS, em que o problema fosse unicamente a qualidade da água, importância total deveria ser dada ao critério da quantidade lançada de efluentes; em contraponto, os pesos da captação $(\alpha)$ e do consumo de água $(\beta)$ seriam nulos. Para esse cenário, os recursos arrecadados dos usuários seriam usados para financiar projetos relacionados com a despoluição das águas. Poderiam ser realizadas, por exemplo, obras de tratamento de esgotos domésticos e controle da poluição industrial.

Em um cenário com essas características, o setor urbano, que é o maior emissor de DBO nas águas da bacia, tornar-se-ia o mais sobrecarregado, respondendo por quase a totalidade dos custos necessários para prover as melhorias ao longo de um ano e pagando a maior taxa entre os usuários, conforme Tabela 6 .

Tabela 6 - Alocação dos custos e taxas cobradas dos usuários, num cenário em que a bacia do rio Paraíba do Sul estivesse altamente poluída

\begin{tabular}{lccc}
\hline Usuário & $\%$ do Custo & $\begin{array}{c}(\%) \times 23.087 .612,82 \\
(\mathrm{R} \$)\end{array}$ & Taxa $\left(\mathrm{R} \$ / \mathrm{m}^{3}\right)$ \\
\hline 1. Indústria & 2,25 & $519.526,61$ & 0,0012 \\
2. Urbano & 97,75 & $22.568 .086,21$ & 0,0398 \\
3. Agrícola & - & - & - \\
\hline Total & 100 & $23.087 .612,82$ & - \\
\hline
\end{tabular}

Fonte: Resultados da pesquisa.

Este cenário seria favorável ao setor agrícola, pois, como este não contribui em nada ou apenas marginalmente para a quantidade lançada de DBO, ele não precisaria arcar com os custos das melhorias. Por sua vez, o setor urbano estaria em situação altamente desfavorável, quando comparado ao cenário da bacia limpa. Uma vez que é o maior lançador 
de DBO na BRPS, este pagaria uma taxa de $0,0398 \mathrm{R} \$ / \mathrm{m}^{3}$, tendo que suportar uma parte equivalente a $97,75 \%$ do custo anual dos projetos a serem implementados. Já o setor industrial, apesar de ser o segundo maior emissor de $\mathrm{DBO}$, não seria tão penalizado, pois pagaria uma taxa de $0,0012 \mathrm{R} \$ / \mathrm{m}^{3}$, o que lhe reservaria uma parcela equivalente a apenas 2,25\% do custo anual dos projetos relacionados à despoluição.

\subsection{Cenário equivalente à bacia do rio Paraíba do Sul $(\alpha+\beta=0,1)$}

Estudos existentes sobre a BRPS ${ }^{16}$ atestam que o maior problema verificado em sua área é o declínio na qualidade da água, decorrente da poluição industrial e, principalmente, dos esgotos urbanos, e que a escassez de água não é um problema imediato, já que ainda há relativa abundância de recursos hídricos na bacia. Com isso, escolheu-se uma ponderação em que o critério do lançamento de efluentes teria peso maior $[(1-\alpha-\beta)=0,9]$ e os critérios das quantidades captada e consumida de água, peso substancialmente menor $(\alpha=\beta=0,05)$, acreditando que esse cenário possa se aproximar da realidade observada na BRPS ${ }^{17}$.

Desse modo, os custos a serem financiados conjuntamente pelos usuários deveriam estar relacionados a múltiplos projetos (que atendessem aos aspectos quantitativo e qualitativo do uso da água), já que o valor de Shapley ponderado considerado nesse cenário levou em conta todos os três critérios. No entanto, projetos de despoluição da bacia poderiam ser apontados como prioritários, dado o maior peso para o lançamento de efluentes. A Tabela 7 apresenta os resultados desse cenário, junto com dados da cobrança existente sobre os usuários, que vigorou de 2003 a 2006 na bacia em questão.

\footnotetext{
${ }^{16}$ Ver, por exemplo, Formiga-Johnsson et al. (2007) e Campos (2001).

${ }^{17}$ Obviamente, num cenário real de aplicação dessa metodologia, a escolha dos pesos deve ser algo a ser discutido amplamente pelos membros participantes dos Comitês de Bacia Hidrográfica, visto que os valores adotados influenciarão diretamente nos resultados.
} 
Tabela 7 - Alocação dos custos e taxas cobradas dos usuários, em um cenário misto, mas considerando elevada poluição na bacia do rio Paraíba do Sul

\begin{tabular}{lccc|cc}
\hline \multirow{2}{*}{ Usuário } & \multicolumn{2}{c|}{ Aplicação do valor de Shapley } & \multicolumn{2}{c}{ Cobrança na BRPS } \\
\cline { 2 - 6 } & Taxa $\left(\mathrm{R} \$ / \mathrm{m}^{3}\right)$ & $\%$ do Custo & $\begin{array}{c}(\%) \times \\
23.087 .612,82 \\
(\mathrm{R} \$)\end{array}$ & $\begin{array}{c}\text { Preço-base } \\
\left(\mathrm{R} \$ / \mathrm{m}^{3}\right)^{*}\end{array}$ & $\begin{array}{c}\% \text { do } \\
\text { Custo }\end{array}$ \\
\hline 1. Indústria & 0,0013 & 2,38 & $549.961,69$ & 0,02 & 35,00 \\
2. Urbano & 0,0360 & 88,40 & $20.409 .161,67$ & 0,02 & 64,00 \\
3. Agrícola & 0,0013 & 9,22 & $2.128 .489,46$ & 0,0005 & 1,00 \\
\hline Total & - & 100 & $23.087 .612,82$ & - & 100 \\
\hline
\end{tabular}

Fonte: Resultados da pesquisa.

*Extraído de Formiga-Johnsson et al. (2007).

O preço-base da cobrança onerava em proporção maior (e em valores iguais) os usuários industriais e urbanos $\left(0,02 \mathrm{R} \$ / \mathrm{m}^{3}\right)$. O setor agrícola, por sua vez, era pouco cobrado, visto que pagava um valor de $0,0005 \mathrm{R} \$ /$ $\mathrm{m}^{3}$, que equivale a apenas $1,25 \%$ do preço dos setores inicialmente citados. No entanto, a taxa encontrada pela aplicação do valor de Shapley visando a alocação do custo anual das melhorias relacionadas a múltiplos projetos teve comportamento diferente, visto que os usuários urbanos pagariam um valor $\left(0,0360 \mathrm{R} \$ / \mathrm{m}^{3}\right)$ bem superior aos que seriam pagos pelos setores industrial e agrícola. Estes últimos arcariam com uma taxa pequena, de igual valor $\left(0,0013 \mathrm{R} \$ / \mathrm{m}^{3}\right)$ e que equivale a somente $3,50 \%$ da taxa cobrada do setor urbano.

A comparação referente aos custos também apresenta pontos dissonantes. Com a taxa proposta neste trabalho, o usuário industrial teria que cobrir apenas $2,38 \%$ do custo e o agrícola arcaria com somente $9,22 \%$ do custo anual das melhorias, enquanto a maior parcela estaria destinada aos usuários urbanos, que teriam que pagar $88,40 \%$ do custo a ser alocado. Essa configuração se distancia do ocorrido na BRPS, pois, de acordo com informações extraídas no site do Comitê de Bacia (CEIVAP, 2008), do total arrecadado até junho de 2006 (ou seja, no 
período de vigência da antiga metodologia de cobrança), $35 \%$ eram oriundos do setor industrial e $64 \%$ do urbano. Já a arrecadação do setor agrícola foi apenas simbólica no mesmo período, já que representou menos de $1 \%$.

Em geral, observando os resultados encontrados nos três cenários apresentados anteriormente, fica claro que a alocação do custo de melhorias entre os setores demandantes é diretamente dependente do critério estabelecido (captação de água, consumo de água, lançamento de efluentes ou, ainda, os três juntos) e, conseqüentemente, do cenário escolhido por meio dos pesos para representar a bacia.

Especificamente, a construção do cenário proposto nesta seção levou em conta os três critérios para criar uma taxa para os usuários de água na BRPS, porém, ao ponderá-los, importância significativa foi dada ao critério da quantidade lançada de efluentes. Os valores encontrados, todavia, mostraram-se distantes dos preços-base que serviam para o cálculo da cobrança que vigorava na bacia, já que o setor urbano seria sobrecarregado, uma vez que sua taxa é bem superior às dos demais, fazendo com que fosse responsável por mais de $88 \%$ do valor a ser alocado entre os três setores demandantes de água considerados ${ }^{18}$.

O valor de Shapley é, por definição, uma taxa justa no sentido de que, como o critério da quantidade lançada de efluentes tem um peso maior neste cenário, o setor que polui em excesso deve, por conseqüência, arcar com a maior parte dos custos dos múltiplos projetos a serem desenvolvidos e pagar o maior valor pelo uso da água. Pode-se depreender que a cobrança na BRPS não seguia este critério de justiça do tipo "quem polui mais paga mais", já que, mesmo sendo responsável por $86,08 \%$ dos efluentes lançados nos cursos d'água na bacia, o setor urbano pagava um preço igual ao do setor industrial $\left(0,02 \mathrm{R} \$ / \mathrm{m}^{3}\right)$, que lançava somente $13,94 \%$ dos efluentes.

\footnotetext{
${ }^{18}$ O setor urbano pagaria valores maiores pela diluição de efluentes, pois o único parâmetro cobrado é a DBO, já que é o único com dados disponíveis. Caso ocorresse a medição de novos parâmetros, o peso da poluição emitida por outros setores certamente aumentaria.
} 


\section{Considerações Finais}

Este trabalho almejou estudar a alocação de custos de melhorias entre os usuários de recursos hídricos em uma bacia hidrográfica, tendo como critérios as quantidades captada e consumida de água e a quantidade lançada de efluentes, representando os aspectos quantitativos e qualitativos do uso da água. Para isso, utilizou-se a regra de alocação conhecida como valor de Shapley, que dá origem a uma taxa cobrada dos diferentes usuários em um período de tempo, visando cobrir o custo das melhorias.

Para que fosse possível levar em consideração os três critérios, simultaneamente, adotou-se um valor de Shapley ponderado, em que a realidade existente na bacia serviu de base para a escolha dos pesos. A aplicação feita na bacia do rio Paraíba do Sul (BRPS) mostrou que o valor de Shapley proposto é extremamente dependente do critério adotado. Para a captação e o consumo de água (bacia limpa), a alocação do custo sobrecarregaria o usuário agrícola, enquanto para o lançamento de efluentes (bacia poluída) maior parte do custo das melhorias seria destinado ao setor urbano.

Quando os três critérios são considerados juntos, todavia, a questão relevante é a escolha correta do peso de cada critério. Como a BRPS é marcada pela poluição de suas águas, significativa importância foi reservada ao lançamento de efluentes. Os valores encontrados foram diferentes dos valores da cobrança na bacia. Com o valor de Shapley, o setor urbano seria sobrecarregado, pagando uma taxa superior aos demais setores e cobrindo parte considerável do valor dos múltiplos projetos.

O valor de Shapley, conforme sua definição, é uma regra de alocação justa já que o principal causador dos custos deverá arcar com a maior parte deles. Como o maior problema da área em estudo é a poluição, o setor que polui mais (urbano) teria que pagar mais. Este resultado é decorrente das características da BRPS. Se o setor urbano tratasse seus esgotos e se as indústrias não controlassem suas emissões de poluição, o 
setor industrial pagaria maiores valores. Ou então, em uma bacia com escassez de recursos hídricos, o peso maior no cálculo do valor de Shapley ponderado seria reservado à captação e ao consumo de água, que afetam esse recurso em seu aspecto quantitativo. Portanto, o setor que mais demanda água (e não o que mais polui) pagaria mais.

Uma possível razão para que a cobrança na BRPS se mostrasse diferente dos resultados encontrados neste trabalho é que os Comitês (que definem a metodologia de cobrança) são órgãos de gestão essencialmente políticos. Em sua formação e nas suas decisões há pressão de grupos de interesse (usuários, Poder Público e até ativistas ambientais), o que pode causar distorções em relação à cobrança baseada nos conceitos de justiça seguidos pelo valor de Shapley.

É importante destacar, novamente, que nos cenários em que se levou em conta a poluição dos corpos hídricos na BRPS, o setor urbano pagaria mais pela diluição de efluentes, pois é o maior emissor de DBO, o único parâmetro poluente com dados disponíveis. Se ocorresse a medição de novos parâmetros ou se a poluição difusa fosse mais facilmente medida, a participação do setor industrial e do agrícola aumentaria, influenciando no resultado final da alocação de custos.

A fórmula apresentada neste trabalho (valor de Shapley) não se restringe apenas à BRPS, mas pode ser aplicada em outras bacias, de acordo com o cenário nelas existente, de modo que a correta relação entre os critérios - representada pelo escolha dos pesos - possa ser considerada. Além disso, é uma fórmula perfeitamente adaptável a outras situações, já que ele possibilita a inclusão de novos jogadores (usuários de água) e a consideração de outros critérios de alocação diferentes dos propostos neste trabalho. 


\section{Referências}

ALIPRANTIS, C.D.; CHAKRABARTI, S.K. Games and decision making. Oxford University Press, New York, 1999. 272 p.

BRASIL. Lei n. 9433, de 8 de janeiro de 1997. Institui a Política de Recursos Hídricos, cria o Sistema Nacional de Gerenciamento de Recursos Hídricos. Diário Oficial da União, Brasília, DF, 9 jan. 1997.

CAMPOS, J.D. Cobrança pelo uso da água nas transposições da bacia do rio Paraíba do Sul envolvendo o setor elétrico. 192 p. Dissertação (Mestrado em Engenharia Civil) - COPPE/UFRJ, Universidade Federal do Rio de Janeiro, Rio de Janeiro, 2001.

CARRERA-FERNANDEZ, J.; FERREIRA, P.M. Otimização dos recursos hídricos em sistemas de bacia hidrográfica: o caso da bacia do Rio Formoso, na Bahia. Revista Econômica do Nordeste, Fortaleza, v. 33, n. 3, p. 536-553, jul-set. 2002.

CEIVAP. Comitê para Integração da Bacia Hidrográfica do Rio Paraíba do Sul. Disponível em < http://www.ceivap.org.br > . Acesso em: 30 ago. 2008.

COPPETEC, Fundação - Laboratório de hidrologia e estudos de meio ambiente. Plano de recursos hídricos da Bacia do Rio Paraíba do Sul. Resende: AGEVAP, 2007. 201p.

FORMIGA-JOHNSSON, R. M.; KUMLER, L.; LEMOS, M.C. The politics of bulk water pricing in Brazil: lessons from the Paraíba do Sul basin. Water Policy, v. 9, p. 87-104, 2007.

LOEHMAN, E.; ORLANDO, J.; TSCHIRHART, J.; WHINSTON, A. Cost allocation for a regional wastewater treatment system. Water Resources Research, vol. 15, n. 2, 193-202, 1979. 
MYERSON, R. B. Game theory: an analysis of conflict. Harvard University Press, Cambridge, 1991. 568 p.

SERRANO, R. Cooperative games: core and shapley value. Department of Economics, Brown University and IMDEA-Social Sciences, 2007. 20 p.

SHAPLEY, L. S. A Value for n-person games. Princeton University Press, p. 307-317, 1953.

TUNDISI, J.G. Ciclo hidrológico e gerenciamento integrado. Ciência e Cultura, São Paulo, v. 55, n. 4, p. 31-33, out./dez. 2003.

VARIAN, H. R. Microeconomic Analysis. 3. ed. New York: W. W. Norton \& Company, 1992. $506 \mathrm{p}$.

YOUNG, H.P.; OKADA, N.; HASHIMOTO, T. Cost allocation in water resources development. Water resources research, vol. 18, n. 3, 463$475,1982$.

Abstract: The objective of this work was to analyze the cost allocation of improvements in river basins among the water users. It was used, as example, the Paraíba do Sul river basin, located in the states of Minas Gerais, São Paulo and Rio de Janeiro, and, as criteria of allocation of costs, they were used the volumes of water extracted and consumed, and the quantity of pollutants deposited in the basin area by water users. The methodology used consists of the rule of allocation known as Shapley value, which belongs to the cooperative branch of game theory. In the case of Paraíba do Sul river basin, the values found indicated that the urban users should pay the higher part of the costs of improvements, given that these users are responsible for most of the pollution in the basin area, being the reduction of water quality an important problem in this basin.

Keywords: water resources, cost allocation, Shapley value, Paraíba do Sul river basin. 
REVISTA DE ECONOMIA E AGRONEGÓCIO, VOL.7, $N^{\circ} 1$ 